\title{
GENERALIZED DIRECT PREDISTORTION WITH ADAPTIVE CREST FACTOR REDUCTION CONTROL
}

\author{
Roberto Piazza, Bhavani Shankar M. R. and Björn Ottersten \\ Interdisciplinary Centre for Security, Reliability and Trust, (SnT), University of Luxembourg
}

\begin{abstract}
Efficient power amplification is inherently a non linear operation that introduces unwanted interference in the amplified signal. Strong inter-symbol interference is generated when the amplifier non linearity is combined with channel memory effects. Further, signals with very high peak to average power ratio, typical of multiple carrier systems, are even more sensitive to the non linearities resulting in severe distortion effects. Signal pre-clipping (crest factor reduction) and predistortion are conventional countermeasure techniques to reduce the generated non linear distortion and improve power and spectral efficiency. In this work, novel optimization methods for predistortion and pre-clipping are analytically derived for a general non-linear communication channel with memory. A combined architecture in which crest factor reduction is followed by signal predistortion is proposed and the parameters are estimated resorting to iterative algorithms based on least squares method. Performance evaluation of the estimation techniques shows the effectiveness of the derived algorithms and significant gain compared to previously known methods.
\end{abstract}

Index Terms - Signal predistortion, Clipping, Volterra, Crest Factor Reduction,Direct Estimation, Least Mean Squares

\section{INTRODUCTION}

Communication systems are based on the assumption that the transmitter can deliver the signal to the receiver with the required level of energy. Power amplification has the key purpose of enhancing the signal power sufficient to compensate for the channel losses and impairments and achieve the target signal to noise ratio at the receiver. However, power amplification is inherently a non-linear operation that introduces distortion of the signal. This distortion is due to the natural saturation effect present in the high power amplifier (HPA) that, depending on the amplitude of input signal distribution and the required power efficiency, can generate significant distortions. Further, the severity of the interference generated is magnified when the non-linearity of the HPA is combined with channel memory effects. A simple mechanism to avoid such distortions is to operate the HPA in the linear region far from the saturation region. However, operating the amplifier in the linear region drastically reduces the power efficiency and the resulting signal output power. The back-off needed for such an operation depends on the peak to average power ratio (PAPR) of the input signal. PAPR refers to the ratio between the peak input power and the average input signal power. Further, the PAPR is significant for multiple carrier signals being amplified by a single HPA.

A common approach to counteract the distortion effect and still maintain the required level of output power, is to perform specific preprocessing of the signal prior to signal amplification. This technique, referred as signal predistortion (DPD) is usually performed on the baseband version of the signal and it is often based on polynomial functions [1, 2, 3, 4, 5] or look up tables (LUT) [6, 7]. While literature mostly focuses on terrestrial applications, signal predistortion techniques are also becoming popular in satellite communications [8] partly due to the move towards amplification of multiple carriers (or multicarrier signals) in a single wideband HPA for reducing the payload mass and mission costs. Very large PAPR values, typical of multicarrier signals, force a substantial component of the signal input distribution beyond the amplifier saturation point. This effect introduces unwanted strong distortion that cannot be compensated with predistortion. Motivated by this, we pursue the study of PAPR reduction in multicarrier systems. Several PAPR reduction methods have been proposed in [9]: amongst these, clipping and filtering can provide significant PAPR reduction with minor interference generation (clipping noise) [10]. Clipping of the signal is in general referred as crest factor reduction (CFR). Further, the use of PAPR reduction in conjunction with signal predistortion has also been widely considered in literature. Several works propose a scheme in which PAPR reduction precedes predistortion [11, 12], while [13] proposes a scheme where predistortion is followed by PAPR reduction. A different approach was recently proposed in [14], that presents a method to include PAPR control as a constraint in the estimation of the predistortion parameters.

In this work we consider a transmitter architecture in which PAPR reduction is performed before signal predistortion. The contribution with respect to the state of the art is two fold: on one hand, we propose a novel optimization framework to automatically determine the optimal level of PAPR reduction towards maximizing system performance; on the other hand, direct estimation for predistortion proposed in [15] is reformulated to include new terms towards improving the parameter optimization and consequently the system performance. While the focus here is on signal predistortion, an optimization of the CFR reduction in presence of data predistortion [16] can be equivalently pursued.

Notation: $\boldsymbol{a}$ and $A$ respectively denote vectors and matrices, $T,{ }^{*}$ denote the transposition and complex conjugation operations, $\otimes$ Kronecker product.

\section{SYSTEM MODEL}

Referring to the Fig. 1, we consider a general baseband multicarrier signal $u(n)$ input to the cascade of CFR and predistortion (DPD) blocks prior to transmission. The channel is assumed to be a nonlinear function with memory.

The general multicarrier signal, $u(n)$, is defined as,

$$
u(n)=\sum_{m} u_{m}(n) e^{j 2 \pi \Delta f_{m} n}
$$

where $\Delta f_{m}$ is the center frequency of $m$ th channel and $u_{m}(n)$ is 


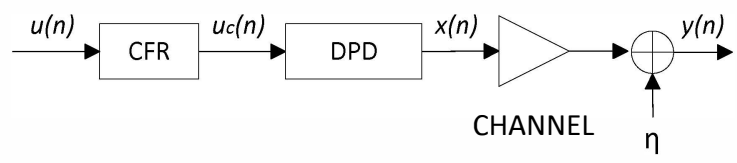

Fig. 1. System Model Block Diagram

the baseband signal of the $m$ th carrier generally defined as,

$$
u_{m}(n)=\sum_{k} a_{m} p_{m}\left(n T_{s}-k T_{m}\right),
$$

where $T_{m}$ is the symbol rate of the $m$ th channel, $T_{s}$ is the oversampling period and $p_{m}(\cdot)$ is the pulse shaping function. Notice that the oversampling period $T_{s}$ is equal for all carriers and such that $T_{s}>>T_{m}$ where $M$ is the total number of carriers. Referring to Fig. $1, u_{c}(n)$ is the signal output of the CFR. The objective of the CFR is to ensure $\operatorname{PAPR}\left(u_{c}(n)\right) \leq \operatorname{PAPR}(u(n))$. Further, $x(n)$, the output of the predistortion block serves as input to the channel.

\subsection{Channel Model}

We consider a general baseband Volterra non-linear system with memory to model the channel [17]. The Volterra baseband model, while being general, it is particularly applicable to satellite channels $[18,19]$. Using Kronecker products, we formulate the general baseband Volterra function in a compact way and referring to Fig. 1, the noiseless channel output is expressed as,

$$
\begin{aligned}
& y(n)=\boldsymbol{h}_{1} G_{1} \boldsymbol{x}_{k_{1}}(n) \\
& +\boldsymbol{h}_{3} G_{3} \boldsymbol{x}_{k_{3}}(n) \otimes \boldsymbol{x}_{k_{3}}(n) \otimes\left[\boldsymbol{x}_{k_{3}}(n)\right]^{*} \\
& +\boldsymbol{h}_{5} G_{5} \boldsymbol{x}_{k_{5}}(n) \otimes \boldsymbol{x}_{k_{5}}(n) \otimes \boldsymbol{x}_{k_{5}}(n)\left[\boldsymbol{x}_{k_{5}}(n) \otimes \boldsymbol{x}_{k_{5}}(n)\right]^{*},
\end{aligned}
$$

where $\boldsymbol{x}_{k_{d}}=\left[x\left(n-k_{d}\right), \cdots, x\left(n+k_{d}\right)\right]^{T}, K_{d}$ is the single side memory relative to the $d$ th degree and $\boldsymbol{h}_{d}$ is a row vector with $L_{d}$ elements where $L_{d}=\prod_{a=0}^{(d-1) / 2}\left(2 k_{d}+1-a\right) \prod_{b=0}^{(d-3) / 2}\left(2 k_{d}+1-b\right)$. $G_{d}$ is a $L_{d} \times\left(2 k_{d}+1\right)^{d}$ matrix selecting the relevant product terms from the $d$ th degree Kronecker product vector for a complete, nonredundant Volterra representation.

In particular we have $G_{3}=\left[\boldsymbol{g}_{i_{1}, j_{1}, k_{1}}^{T}, \cdots, \boldsymbol{g}_{i_{L_{3}}, j_{L_{3}}, q_{L_{3}}}^{T}\right]^{T}$ where each row vector corresponds to a standard basis $\boldsymbol{g}_{i_{s}, j_{s}, q_{s}}=$ $\boldsymbol{e}_{p}$ with $p=q_{s}+\left(j_{s}-1\right)\left(2 k_{3}+1\right)+\left(i_{s}-1\right)\left(2 k_{3}+1\right)^{2}$. Further, we have $\left(i_{s}, j_{s}, q_{s}\right) \in \Omega_{3}$ where $\Omega_{3}=\{(i, j, q) \mid \forall i \in$ $\left.\left(1, \cdots, 2 k_{3}+1\right), \forall j \in\left(i, \cdots, 2 k_{3}+1\right), \forall q \in\left(1, \cdots, 2 k_{3}+1\right)\right\}$. Similarly, $G_{5}=\left[\boldsymbol{g}_{i_{1}, j_{1}, q_{1}, a_{1}, b_{1}}^{T}, \cdots, \boldsymbol{g}_{i_{L_{5}}, j_{L_{5}}, q_{L_{5}}, a_{L_{5}}, b_{L_{5}}}^{T}\right]^{T}$ where each row vector corresponds to a standard basis $\boldsymbol{g}_{i_{s}, j_{s}, q_{s}, a_{s}, b_{s}}=$ $\boldsymbol{e}_{p}$ with $p=b_{s}+\left(a_{s}-1\right)\left(2 k_{5}+1\right)+\left(q_{s}-1\right)\left(2 k_{5}+1\right)^{2}+$ $\left(j_{s}-1\right)\left(2 k_{5}+1\right)^{3}+\left(i_{s}-1\right)\left(2 k_{5}+1\right)^{4}$. Further, we have $\left(i_{s}, j_{s}, q_{s}, a_{s}, b_{s}\right) \in \Omega_{5}$ where $\Omega_{5}=\left\{(i, j, q, a, b) \mid \forall i \in\left(1, \cdots, 2 k_{5}+\right.\right.$ $1), \forall j \in\left(i, \cdots, 2 k_{5}+1\right), \forall q \in\left(j, \cdots, 2 k_{5}+1\right), \forall a \in\left(1, \cdots, 2 k_{5}+\right.$ 1), $\left.\forall b \in\left(a, \cdots, 2 k_{5}+1\right)\right\}$.

\subsection{Crest Factor Reduction Model}

The output of CFR is related to its input as

$$
u_{c}(n)= \begin{cases}u(n) & \text { if }|u(n)| \leq|\gamma|^{2} \\ |\gamma|^{2} u(n) /|u(n)| & \text { if }|u(n)|>|\gamma|^{2}\end{cases}
$$

where $\gamma$ is the clipping parameter. Notice that the CFR clipping function does not modify the phase of the complex signal, i. e. $\angle u_{c}(n)=\angle u(n)$. Further, the clipping function as defined here, is slightly different from the standard clipping function of [20] and involves $|\gamma|^{2}$ rather than $\gamma$. This choice will enable the formulation of the optimization algorithm to compute the optimal clipping value.

\subsection{Predistorter Model}

The predistortion function acts on the output of the clipping function to yield the channel input $x(n)$. The predistorter per-se is a non-linear function with memory accomodating various polynomial functions including memory polynomials [1], generalized memory polynomials [3] and Volterra expansion [5]. The output of the predistorter is computed as,

$$
x(n)=[\phi(n)]^{T} \boldsymbol{w},
$$

where the predistorter function is explicitly expressed as a function of the parameter $m \times 1$ vector $\boldsymbol{w}$ and where $\phi(n)$ is a $m \times 1$ vector collecting the linear and non-linear terms generated from input signal $u_{c}(n)$ including linear terms $\{u(n-k)\}$ third degree terms $\left\{u\left(n-k_{1}\right) u\left(n-k_{2}\right) u^{*}\left(n-k_{3}\right)\right\}$ and so on $[1,5]$. The value of $m$ depends on the degree and the associated memory depth $[1,5]$ and is not detailed further for ease of comprehension. Central to the performance of the predistortion is the determination of the parameters $\boldsymbol{w}$. Well known methods for estimation of predistortion parameters are the indirect [5] and direct learning [15] approaches.

Referring to Fig. 1 we notice that the DPD block is between the CFR block and the channel. This means that the predistortion function is not designed to invert the channel function but rather to reduce the receiver interference. This fundamental observation suggests a direct estimation approach rather then an indirect one [21] for obtaining $\boldsymbol{w}$.

\section{ADAPTIVE PEAK CONTROLLED DIRECT PREDISTORTION}

Given the crest factor reduction and predistortion techniques proposed in Sections 2.2 and 2.3, we define a transmitter architecture in which the CFR block precedes the DPD block. In this section, we derive optimization algorithms to determine the optimal CFR and predistortion parameters towards reducing the error at the receiver. Fig. 2 illustrates the considered transmitter architecture including the two iterative optimization processes.

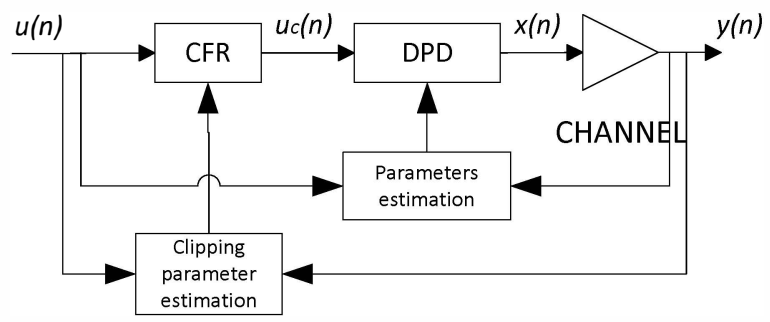

Fig. 2. Combined Optimization Function Block Diagram 


\subsection{Generalized Direct Estimation}

The target of the predistortion function is to reduce the non-linear distortion at the channel output. This is achieved by optimizing the parameters $\boldsymbol{w}$. The general estimation paradigm is shown in Fig. 2 .

Referring to (3) and (5), we define

$$
\begin{aligned}
& \boldsymbol{x}_{k_{i}}(n)=\Phi_{k_{i}}(n) \boldsymbol{w}, \\
& \Phi_{k_{i}}=\left[\phi\left(n-k_{i}\right), \cdots, \phi\left(n+k_{i}\right)\right]^{T} .
\end{aligned}
$$

Combining (3) with (6) and limiting the expansion to third degree polynomial for ease of presentation, we obtain,

$$
\begin{aligned}
y(n)= & \boldsymbol{h}_{1} G_{1} \Phi_{k_{1}}(n) \boldsymbol{w} \\
& +\boldsymbol{h}_{3} G_{3}\left[\Phi_{k_{3}}(n) \otimes \Phi_{k_{3}}(n) \otimes \Phi_{k_{3}}(n)^{*}\right]\left[\boldsymbol{w} \otimes \boldsymbol{w} \otimes \boldsymbol{w}^{*}\right] .
\end{aligned}
$$

Similarly to [15], we define the cost function for the optimization of the predistortion parameters as $C(\boldsymbol{w})=|u(n)-y(n)|^{2}$ and formulate a least mean squares (LMS) algorithm for minimizing $C(\cdot)$. We can then derive the update equation for $\boldsymbol{w}$ as,

$$
\boldsymbol{w}(n+1)=\boldsymbol{w}(n)-\mu \frac{\partial C(\boldsymbol{w})}{\partial \boldsymbol{w}}
$$

where $\mu$ is the algorithm step. Using $C(\mathbf{w})$ in (9), we obtain

$$
\boldsymbol{w}(n+1)=\boldsymbol{w}(n)+\mu\left(e^{*}(n) \frac{\partial y(n)}{\partial \boldsymbol{w}(n)}+e(n) \frac{\partial y^{*}(n)}{\partial \boldsymbol{w}(n)}\right) .
$$

where $e(n)=u(n)-y(n)$, and

$$
\begin{aligned}
\frac{\partial y(n)}{\partial \boldsymbol{w}}= & \boldsymbol{h}_{1} G_{1} \Phi_{k_{1}}(n) \\
& +\boldsymbol{h}_{3} G_{3}\left[\Phi_{k_{3}}(n) \otimes \Phi_{k_{3}}(n) \otimes \Phi_{k_{3}}(n)^{*}\right] \\
& {\left[\boldsymbol{w} \otimes I_{m}+I_{m} \otimes \boldsymbol{w}\right] \otimes \boldsymbol{w}^{*}, } \\
\frac{\partial y^{*}(n)}{\partial \boldsymbol{w}}= & \boldsymbol{h}_{3}^{*} G_{3}^{*}\left[\Phi_{k_{3}}^{*}(n) \otimes \Phi_{k_{3}}^{*}(n) \otimes \Phi_{k_{3}}(n)\right] \\
& \boldsymbol{w}^{*} \otimes \boldsymbol{w}^{*} \otimes I_{m},
\end{aligned}
$$

where $I_{m}$ is an $m \times m$ identity matrix (recall from Section 2.3 that the size of $\boldsymbol{w}$ is $m \times 1$ ). Notice that in the derivation of the direct estimation method in [15], the term in (12) is not included in the algorithm. This results in a performance degradation which is illustrated in Section 4. In order to guarantee optimal performance, the vector of predistortion parameters, $\boldsymbol{w}$, is initialized with the result of the off-line indirect estimation [22] where CFR is assumed to be absent.

\subsection{Adaptive Crest Factor Reduction}

The CFR block performs clipping of the original signal before signal predistortion. The challenge of signal clipping is to understand if and to what extent it is convenient to clip the signal. In fact, clipping too much results in a loss of the signal while not clipping would naturally enhance PAPR. In particular, referring to (4), it is necessary to estimate the parameter $\gamma$ towards improving the system performance.

The cascaded predistortion and channel blocks (kindly refer to Figs. 1,2) result in a non-linear system with memory that can be modeled using a Volterra system as (here limited to the third degree),

$$
\begin{aligned}
& y(n)=\sum_{k}^{K_{1}} q^{(1)}(k) u_{c}(n-k) \\
& +\sum_{k_{1}, k_{2}, k_{3}}^{K_{3}} q^{(3)}\left(k_{1}, k_{2}, k_{3}\right) u_{c}\left(n-k_{1}\right) u_{c}\left(n-k_{2}\right) u_{c}^{*}\left(n-k_{3}\right)
\end{aligned}
$$

where $K_{1}$ quantifies the linear memory, $K_{3}$ is the third degree memory component and $\left\{q^{(d)}(\cdot)\right\}$ are the model parameters relative to the $d$ th degree. Parameters in (13) can be estimated during on-line operation using standard least squares techniques [23]. Towards this, we consider the cost function $C(\gamma)=|u(n)-y(n)|^{2}$ and formulate a least mean squares algorithm to determine the optimal $\gamma$. We define the update equation for $\gamma$ as,

$$
\gamma(n+1)=\gamma(n)-\epsilon \frac{\partial C(\gamma)}{\partial \gamma}
$$

where $\epsilon$ is the algorithm step. Notice that, in general, we have $\gamma \in \mathbb{C}$. From (14), and using the chain rule, we obtain,

$$
\begin{aligned}
& \gamma(n+1)=\gamma(n)+ \\
& \epsilon\left[e(n)^{*} \frac{\partial y(n)}{\partial u_{c}(n)} \frac{\partial u_{c}(n)}{\partial \gamma}+e(n) \frac{\partial y^{*}(n)}{\partial u_{c}(n)} \frac{\partial u_{c}(n)}{\partial \gamma}\right]
\end{aligned}
$$

where $e(n)=u(n)-y(n)$ and both $\frac{\partial y(n)}{\partial u_{c}(n)}$ and $\frac{\partial y^{*}(n)}{\partial u_{c}(n)}$ can be derived analytically from (13) using the partial differential rules provided in [24]. Notice that, in general, $\frac{\partial y^{*}(n)}{\partial u_{c}(n)} \neq\left[\frac{\partial y(n)}{\partial u_{c}(n)}\right]^{*}$. Further, we can define analytically the differential quantity

$$
\frac{\partial u_{c}(n)}{\partial \gamma}= \begin{cases}0 & \text { if }|u(n)|<|\gamma|^{2} \\ \gamma^{*} e^{i \angle u(n)} & \text { if }|u(n)|>|\gamma|^{2}\end{cases}
$$

where we used $\frac{\partial|\gamma|^{2}}{\partial \gamma}=\gamma^{*}$ [24]. Notice that for $|u(n)|=|\gamma|^{2}$, the derivative of (16) does not exist and hence its value is set to 0 in the simulations. Further, if the algorithm is initialized such that $\left|\gamma_{\text {init }}\right|^{2}>\max |u(n)|$ the resulting derivative will always be zero and the algorithm will only produce $|\gamma(n)|^{2}=\left|\gamma_{\text {init }}\right|^{2} \forall n$. Therefore, key to the convergence of the algorithm, is the choice of the initial guess $\gamma_{\text {init }}$. This can be trivially set to 0 or obtained from knowing the amplifier output saturation power, the signal PAPR and the selected Input Backoff $\left(\mathrm{IBO}=10 \log _{10}\left(P_{i n} / P_{i n}^{\mathrm{SAT}}\right)\right.$, where $P_{i n}$ is the amplifier signal input power and $P_{i n}^{S A T}$ is the amplifier saturation input power).

\section{NUMERICAL RESULTS}

In this section we numerically evaluate the performance of the algorithms for a selected case of study.

\subsection{Simulation Scenario}

We consider a typical satellite communication scenario where the channel includes a satellite transponder model [18]. As transponder model, we consider an input multiplexer filter (IMUX), a HPA, and an output multiplexer filter (OMUX). Typical responses for the IMUX and OMUX filter are extracted from [25] and modelled as FIR filters. On the other hand, the HPA is parameterized using the standard Saleh model with AM/AM and AM/PM functions taking the form $A(r)=\frac{2 r}{1+r^{2}} ; \Phi(r)=\frac{\pi}{6} \frac{r^{2}}{1+r^{2}}$, respectively [16].

The channel input signal $u(n)$ consists of five independent DVB-S2 [26] carriers, each employing 16APSK modulation, with identical symbol rate $R_{s}$ and spaced equally in frequency with a separation of $R_{s}(1+\rho)$ where $\rho=0.2$ is the pulse roll off. The resulting PAPR of the signal, without compensation, is $9.8 \mathrm{~dB}$. 


\subsection{Algorithm Setting}

Both estimation algorithms described in Sections 2.2 and 3.1 are trained over 100000 samples corresponding to 10000 symbols per carrier (oversampling factor or 10). Step parameters are determined by fine simulation tuning to be: $\epsilon=10^{-2}$ in (16); $\mu=10^{-5}$ in (10). Notice that, in all considered cases, training is carried out in absence of receiver noise. The channel parameters $\mathbf{h}_{d}$ (kindly refer to (3) with $k_{1}=5, k_{3}=3$ and $k_{5}=0$ ) and the parameters corresponding to the cascaded predistortion and channel function $\left(q^{(d)}(\cdot)\right.$ in (13) with $K_{1}=3, K_{3}=2$ and $K_{5}=1$ ), are estimated on-line using standard least squares (LS) techniques [23].

The predistortion function of (5) implements a standard memory polynomial [1] of fifth degree with single side memory depth $k_{1}=$ $5, k_{3}=3$ and $k_{5}=1$ for each degree respectively.

Further, in the case when both CFR and DPD algorithms are applied, they can be run alternately or concurrently. However, when running concurrently the final performance is slightly penalized due to the inter dependency in the target error. For the sake of performance optimization, we alternate them in three phases: we first perform a DPD estimation followed by the CFR estimation and a subsequent DPD estimation. We noticed that further iterations do not improve significantly the performance.

Further, depending on the channel characteristics and the parameter setting, the convergence property of the proposed algorithms might vary. In general, we observed poorer convergence when the channel exhibits longer memory effects.

\subsection{Performance Evaluation}

\subsubsection{Figure of Merit}

As figure of merit, we consider the signal to interference plus noise ratio (SINR) at the output of the channel with respect to the amplifier output backoff $\left(\mathrm{OBO}=10 \log _{10}\left(P_{\text {out }} / P_{\text {out }}^{\mathrm{SAT}}\right)\right.$, where $P_{\text {out }}$ is the output power of the amplified signal and $P_{\text {out }}^{\mathrm{SAT}}$ is the amplifier saturation output power). In practice, the OBO determines the operating point of the amplifier and hence determines both the useful output power and amount on non-linearities. For the current study, we focus on the impact of $\mathrm{OBO}$ on non-linearities; in particular, the receiver signal to noise ratio (SNR) is fixed. Further, we plot the power spectral densities at the channel output to observe and compare the spectral regrowth. Towards quantifying the spectral shaping, we also consider the normalized mean square error (NMSE) at the receiver in absence of noise as NMSE $=1 / N \sum_{n=1}^{N}|u(n)-y(n)|^{2} / \sum_{n=1}^{N}|y(n)|^{2}$ computed with respect the channel output signal $y(n)$ with respect to the properly delayed version of the input signal $u(n)$.

\subsubsection{SINR Performance}

Fig. 3 depicts the SINR versus OBO for different techniques with a set $\mathrm{SNR}=20 \mathrm{~dB}$. Referring to Fig. 3 and focussing on the cases in which CFR is not applied, we present three sets of results: standard indirect estimation [4] (brown), conventional direct estimation [15] (red) and our novel direct estimation algorithm (green) of Section 3.1. Observing Fig. 3, we have that standard indirect predistortion and conventional direct predistortion have similar performance while the reformulated LMS algorithm of (10) provides additional performance gain over [15] of about $1-1.25 \mathrm{~dB}$ due to inclusion of new terms. Considering the combination of CFR and DPD, we compare the peak constraint predistortion of [12] with our method. Referring to Fig. 3 we have a significant gain in performance of our proposed method over [12] of about $2 \mathrm{~dB}$.

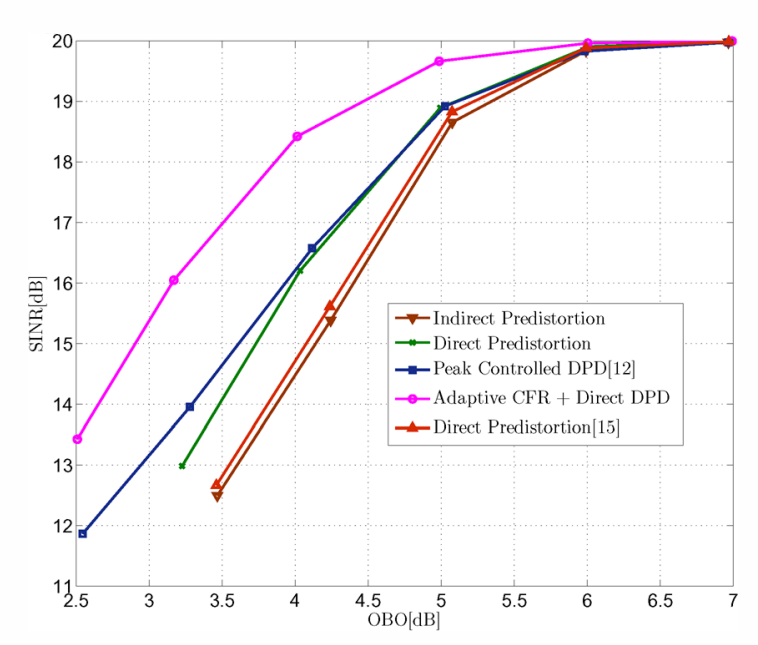

Fig. 3. Signal to Interference plus Noise versus Output Back-Off

\subsubsection{Spectral Density and NMSE}

At the output of the channel (output of the OMUX filter), we can observe the typical spectral regrowth of the signal due to the nonlinear characteristic of the amplifier. In Fig. 4 we can observe the power spectral density of the multicarrier signal (only the positive part it is shown) for the different techniques considered.

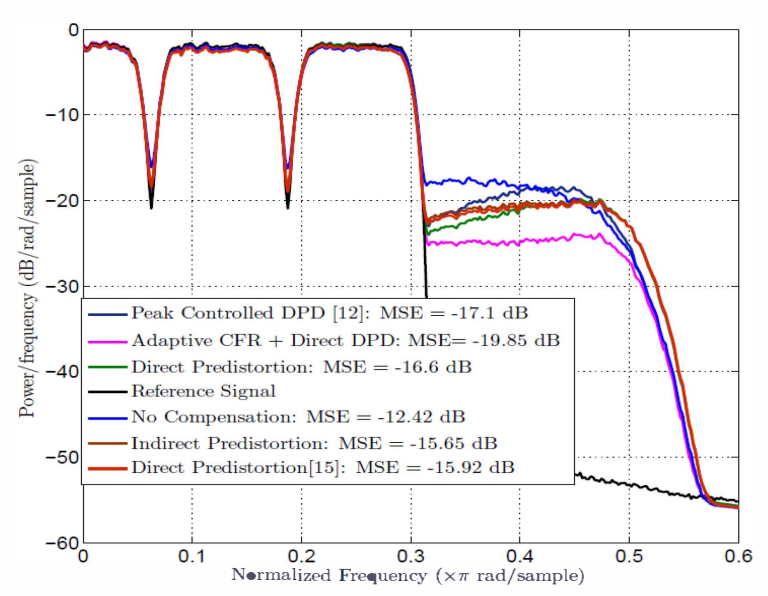

Fig. 4. Power Spectral Density Response for Five Carriers DVB-S2 carriers at $\mathrm{IBO}=4 \mathrm{~dB}$

As expected, techniques with a lower NMSE also show a lower spectral regrowth.

\section{CONCLUSION}

A novel design method for combined CFR and DPD has been proposed. This includes an automatic method to determine the optimal clipping amplitude for a general non-linear channel with memory together with a reformulated and generalized direct estimation method for predistortion. While the reformulated direct estimation itself shows to provide gain with respect to the state of the art, the combination of CFR and DPD is shown to provide the best performance. 


\section{REFERENCES}

[1] Lei Ding, G. T Zhou, D. R Morgan, Zhengxiang Ma, J. S. Kenney, Jaehyeong Kim, and C. R. Giardina, "A robust digital baseband predistorter constructed using memory polynomials," IEEE Trans. Commun., vol. 52, no. 1, pp. 159-165, Jan. 2004.

[2] R. Raich, Hua Qian, and G.T. Zhou, "Orthogonal polynomials for power amplifier modeling and predistorter design," IEEE Trans. Veh. Technol., vol. 53, no. 5, pp. 1468 - 1479, Sept. 2004.

[3] D.R. Morgan, Zhengxiang Ma, Jaehyeong Kim, M.G. Zierdt, and J. Pastalan, "A generalized memory polynomial model for digital predistortion of RF power amplifiers," Signal Processing, IEEE Transactions on, vol. 54, no. 10, pp. 3852-3860, Oct 2006.

[4] Lei Ding, Raviv Raich, and G. Tong Zhou, "A Hammerstein predistortion linearization design based on the indirect learning architecture," in Acoustics, Speech, and Signal Processing (ICASSP), 2002 IEEE International Conference on, May 2002, vol. 3, pp. III-2689-III-2692.

[5] Changsoo Eun and E.J. Powers, "A new Volterra predistorter based on the indirect learning architecture," Signal Processing, IEEE Trans. on, vol. 45, no. 1, pp. 223 -227, Jan 1997.

[6] K.J. Muhonen, Mohsen Kavehrad, and R. Krishnamoorthy, "Look-up table techniques for adaptive digital predistortion: a development and comparison," Vehicular Technology, IEEE Transactions on, vol. 49, no. 5, pp. 1995-2002, Sep 2000.

[7] Bo Ai, Zhi-Xing Yang, Chang-Yong Pan, Shi gang Tang, and Tao tao Zhang, "Analysis on LUT based predistortion method for HPA with memory," Broadcasting, IEEE Transactions on, vol. 53, no. 1, pp. 127-131, March 2007.

[8] Thomas J. Brazil Noel Kelly, Anding Zhu, “Digital predistortion feasibility studies for multicarrier satelite communication systems," in Proc. 31th AIAA Intern.Commun. Satellite Syst. Conference (ICSSC), Florence, Italy, Oct. 2013.

[9] Seung Hee Han and Jae Hong Lee, "An overview of peak-toaverage power ratio reduction techniques for multicarrier transmission," Wireless Communications, IEEE, vol. 12, no. 2, pp. 56-65, April 2005.

[10] Dukhyun Kim and G.L. Stuber, "Clipping noise mitigation for OFDM by decision-aided reconstruction," Communications Letters, IEEE, vol. 3, no. 1, pp. 4-6, Jan 1999.

[11] O.A Gouba and Y. Louet, "Joint study of PAPR reduction and digital predistortion," in General Assembly and Scientific Symposium, 2011 XXXth URSI, Aug 2011, pp. 1-4.

[12] C. Nader, P.N. Landin, W. Van Moer, N. Bjorsell, P. Handel, and D. Ronnow, "Peak-power controlling technique for enhancing digital pre-distortion of RF power amplifiers," $\mathrm{Mi}$ crowave Theory and Techniques, IEEE Transactions on, vol. 60, no. 11, pp. 3571-3581, Nov 2012.

[13] R.N. Braithwaite, "A combined approach to digital predistortion and crest factor reduction for the linearization of an RF power amplifier," Microwave Theory and Techniques, IEEE Transactions on, vol. 61, no. 1, pp. 291-302, Jan 2013.

[14] P.N. Landin, W. Van Moer, M. Isaksson, and P. Handel, "Peakpower controlled digital predistorters for RF power amplifiers," Microwave Theory and Techniques, IEEE Transactions on, vol. 60, no. 11, pp. 3582-3590, Nov 2012.
[15] D. Zhou and V. E. DeBrunner, "Novel adaptive nonlinear predistorters based on the direct learning algorithm," Signal Processing, IEEE Transactions on, vol. 55, no. 1, pp. 120 -133, Jan. 2007.

[16] R. Piazza, B. Shankar, and B. Ottersten, "Data predistortion for multicarrier satellite channels based on direct learning," submitted to Signal Processing, IEEE Transactions on, Oct. 2013.

[17] Martin Schetzen, The Volterra and Wiener Theories of Nonlinear Systems, John Wiley \& Sons, Apr. 1980.

[18] Giovanni E. Corazza, Digital Satellite Communications, Chapter 8, Springer, 2007.

[19] S Benedetto and E Biglieri, "Nonlinear equalization of digital satellite channels," IEEE J. Sel. Areas Commun., vol. 1, pp. 57 -62, Jan. 1983.

[20] J. Armstrong, "Peak-to-average power reduction for OFDM by repeated clipping and frequency domain filtering," Electronics Letters, vol. 38, no. 5, pp. 246-247, Feb 2002.

[21] Mazen Abi Hussein, Vivek Ashok Bohara, and Olivier Venard, "On the system level convergence of ILA and DLA for digital predistortion," in Wireless Communication Systems (ISWCS), 2012 International Symposium on, Aug 2012, pp. 870 -874.

[22] M.A. Borkar, F.A. Mujica, and G. Copeland, "Method and system for calculating the pre-inverse of a nonlinear system," June 8 2010, US Patent 7,733,177.

[23] V. John Mathews, Polynomial Signal Processing, New York: Wiley, 2000.

[24] Monson H. Hayes, Statistical Digital Signal Processing and Modeling, New York:Wiley, 1998.

[25] E. Casini, R. De Gaudenzi, and A. Ginesi, "DVB- S2 modem algorithms design and performance over typical satellite channels," Intern. J.on Satellite Commun. and Networking, vol. 22, pp. 281-318, 2004.

[26] "Digital video broadcasting (DVB); second generation framing structure, channel coding and modulation systems for broadcasting, interactive services, news gathering and other broadband satellite applications (DVB-S2)," 2012. 